\title{
Silent ischemic brain lesions detected by multi-slice computed tomography are associated with subclinical atrial fibrillation in patients with cardiac resynchronization therapy
}

\author{
Yahya Kemal Icen ${ }^{1}$, Ayse Selcan Koc ${ }^{2}$ \\ ${ }^{1}$ Department of Cardiology, Adana Health Practices and Research Center, Health Sciences University, Adana, Turkey \\ ${ }^{2}$ Department of Radiology, Adana Health Practices and Research Center, University of Health Sciences, Adana, Turkey
}

Adv Interv Cardiol 2018; 14, 3 (53): 285-290

DOI: https://doi.org/10.5114/aic.2018.78332

\begin{abstract}
A bstract
Introduction: There is insufficient research on the relationship between subclinical atrial fibrillation (SCAF) and silent ischemic brain lesions (IBLS).

Aim: To investigate the relationship between SCAF and silent IBLs in patients with cardiac resynchronization therapy (CRT).

Material and methods: Of 720 CRT implanted patients in our department between 2012 and 2018, 121 patients who underwent elective cranial multi-slice computed tomography (MSCT) during their follow-up were included in our study. Atrial high-rate episodes (AHRE) were detected by the CRT device. Subclinical atrial fibrillation was defined as asymptomatic AHRE longer than $6 \mathrm{~min}$ and shorter than $24 \mathrm{~h}$. A cranial MSCT scan was performed using a 128-section scanner with contiguous $2-5 \mathrm{~mm}$ axial images. Patients were divided into two groups - with and without silent IBL.

Results: Silent IBLs were detected in $21(17.4 \%)$ of 121 patients with CRT. Ischemic brain lesion presence was found to be associated with age, CHA2DS2-VASc score, left ventricular (LV) ejection fraction (EF), hypertension and SCAF in univariate analysis $(p<0.05)$. In multivariate regression analysis, presence of SCAF and LVEF were found to be independent parameters predicting the risk of silent IBLs. According to this analysis, the presence of SCAF and every $1 \%$ decrease in LVEF were found to increase the risk of silent IBL by 3.5 times and $14.8 \%$, respectively.

Conclusions: Subclinical atrial fibrillation is independently associated with silent IBL presence. Patients with CRT should be closely monitored for SCAF. Patients diagnosed with SCAF should be evaluated for IBL development and treated with the appropriate oral anticoagulant.
\end{abstract}

Key words: cardiac resynchronization therapy, brain ischemia, atrial fibrillation.

S u m m a ry

Subclinical atrial fibrillation can be detected with cardiac devices and may cause ischemic brain lesions. These lesions may affect the mortality and morbidity of the patient. For this reason, early detection of these patients is important.

\section{Introduction}

Atrial fibrillation $(\mathrm{AF})$ is the most common arrhythmia in patients with heart failure (HF). Studies have shown that $\mathrm{HF}$ increased the risk of developing AF by $43 \%$ [1]. Because of this association, detection of clinical AF, subclinical AF (SCAF) and silent $A F$ is of great importance in $\mathrm{HF}$ patients to prevent thromboembolic events. In patients with cardiac resynchronization therapy (CRT), continuous recordings of the atrial lead in place of conventional methods (Holter, ECG, etc.) made it possible to detect SCAF with ease of access and were included in the 2016 ESC AF guideline [1]. In the studies that evaluate the relationship between the presence of atrial high-rate episodes (AHRE)

\section{Corresponding author:}

Koc Ayse Selcan MD, Department of Radiology, Adana Health Practices and Research Center, University of Health Sciences,

Dr. Mithat Özsan Bulvarı Kışla Mah. 4522 Sok. No: 1, 01370 Yüreğir/ADANA Adana, Turkey, phone: +90 5062425988, fax: +90 322 455 90 00,

e-mail: drayseselcankoc@gmail.com

Received: 29.03.2018, accepted: 17.06.2018. 
and AF in CRT patients and their clinical significance and association with thromboembolic events, AHRE and AF were found to increase the risk of stroke 2-5-fold [2-4]. In the study of Shanmugam et al. the risk of thromboembolic events was four times higher in CRT patients with AHRE. Although the relationship between SCAF and clinical stroke is clearly demonstrated in the literature, the relationship between SCAF and silent ischemic brain lesions (IBL) has not been evaluated in patients with CRT. Only Benezet-Mazuecos et al. [5] showed a close and independent relationship between AHRE > 5 min and IBL in patients carrying cardiac devices [5].

In our study, we aimed to investigate the relationship between SCAF and silent IBL detected with cranial multislice computed tomography (MSCT) in patients with CRT.

\section{Aim}

The aim of this study to investigate the relationship between SCAF and silent IBL in patients with CRT.

\section{Material and methods}

\section{Study population}

Of 720 CRT implanted patients in our department between 2012 and 2018, 132 patients who underwent elective cranial MSCT during their follow-up were included in our retrospective study. Patients with previous history of documented AF, presence of related symptoms suggesting $A F$, respiratory diseases (chronic obstructive pulmonary disease, chronic bronchitis, pulmonary embolism), primary pulmonary hypertension, prior or acute stroke or transient ischemic attack, acute coronary syndrome in the last 2 months, isolated right HF, congenital heart disease, or severe valve disease (aortic stenosis, mitral stenosis and regurgitation) were excluded from the study.

After the exclusion criteria were applied, the study included 121 patients (54 male, 67 female; mean age: $65.6 \pm 7.8$ years). The Local Ethics Committee approved the study protocol and each participant provided written informed consent.

After assessment of detailed medical history and a complete physical examination, the baseline characteristics of patients including age, sex, history of ischemic heart disease, dilate cardiomyopathy, hypertension, diabetes, hyperlipidemia, current smoking status and medications were recorded for all patients. All patients had QRS duration greater than 120 msn. Patients were classified according to the NYHA staging system by two cardiologists. If there was no consensus, NYHA class was determined by a joint decision including a third cardiologist. Weight and height were taken and body mass indexes were calculated. $\mathrm{CHA}_{2} \mathrm{DS}_{2}$-VASc scores and HAS-BLEED scores were calculated. Patients' medical treatment was adjusted according to recent HF guidelines and their clinical stage.

\section{Echocardiographic and electrocardiographic evaluation}

Baseline ECGs of patients were recorded (Nihon Kohden, Cardiofax V, model ECG-1550K, $25 \mathrm{~mm} / \mathrm{s}$ rate and $1 \mathrm{mV} / 10 \mathrm{~mm}$ standard) and heart rhythm (sinus, AF), left branch block and QRS durations were calculated. Left ventricular (LV) ejection fraction (EF) and left atrial (LA) end-diastolic diameter were obtained from the hospital records by accessing the previous echocardiographic measurements (Phillips Healthcare, DA Best, Netherlands).

\section{Pacemaker measurements}

The examination of AT/AF episodes was carried out in the routine follow-up of patients and recorded. Pacemaker measurements were made with a MEDTRONIC Programmer 2090 programmable pacemaker analyzer. Firstly, patients' AT/AF episodes were examined from the device logs (interrogation window). Secondly, the records of all patients diagnosed with AT/AF episodes were reviewed by two experienced cardiologists. After that, these patients with AF were included in our study (Figure 1). In recent studies $\geq 6$ min was taken as a criterion for $\operatorname{SCAF}[6,7]$. As a result of this study and other findings, the baseline AHRE duration for the SCAF definition in the last published consensus was increased from 5 min to 6 min [8]. Intracardiac ECGs were obtained at the time of the event when the device detected SCAF between $6 \mathrm{~min}$ and $24 \mathrm{~h}$. The documented SCAF episodes were placed in patient files.

\section{Cranial multi-slice computed tomography scan procedure}

Magnetic resonance imaging (MRI) is superior to MSCT in assessing IBL. Magnetic resonance imaging can be used with MRI-conditional cardiovascular implantable electronic devices [8]. However, none of our patients had an MRI-conditional device. Therefore, cranial CT was used for imaging. The cranial CT scan was performed using the 128-section scanner (Philips CT Ingenuity 128), with contiguous $2-5 \mathrm{~mm}$ axial images. Two radiologists examined the MSCT scans independently. Ischemic brain infarction is defined as a focal lesion of the same intensity as the cerebrospinal fluid in MSCT. After the MSCT scan, the diameter of the low-density area was measured. The IBLS were defined as lesions $\geq 3 \mathrm{~mm}$ separated by a sharp border from surrounding tissue (Figure 2). Patients with prior or acute stroke or TIA were excluded from our study. For this reason, all detected IBLs were considered as silent infarcts.

\section{Statistical analysis}

Statistical analyses were conducted using SPSS, version 20.0, (SPSS Inc. Chicago, Illinois). Data are expressed 


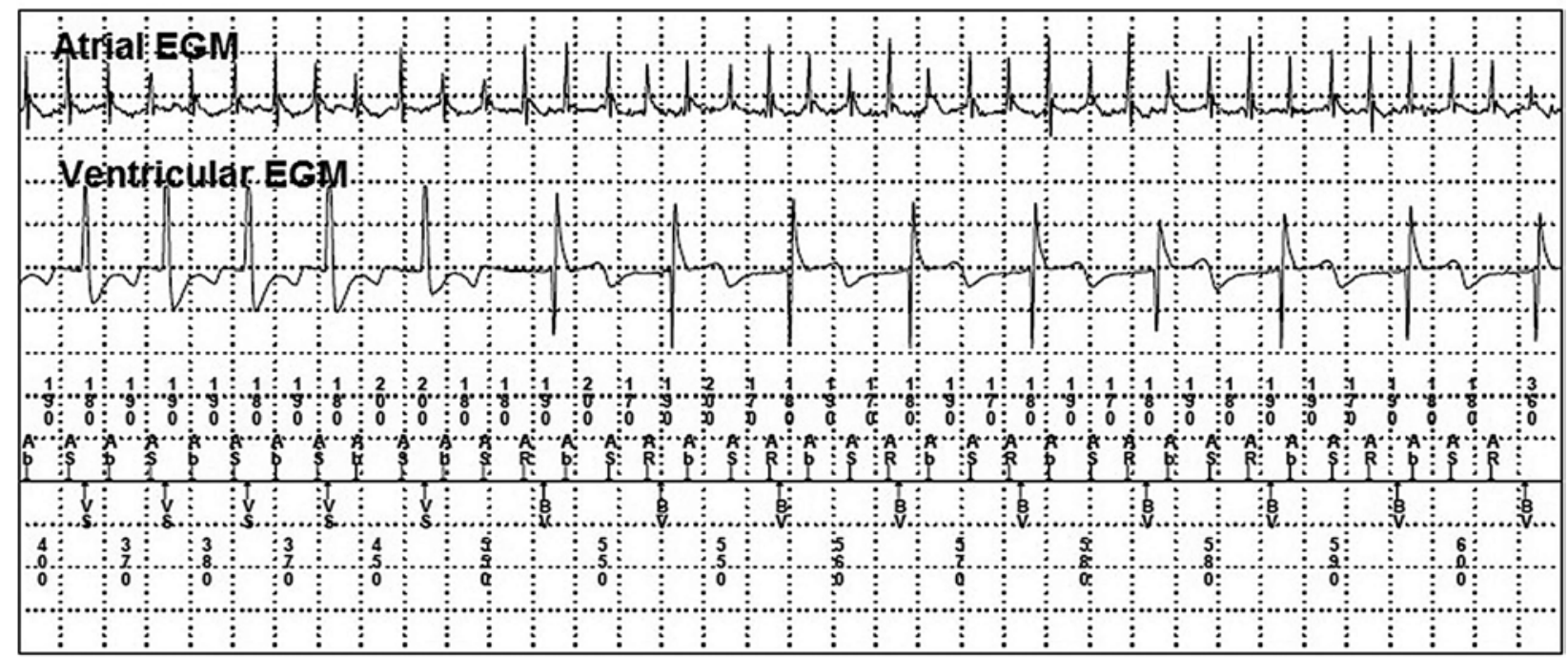

Figure 1. Demonstration of atrial fibrillation episode with MEDTRONIC programmer 2090 device in patients with Protecta CRT-D
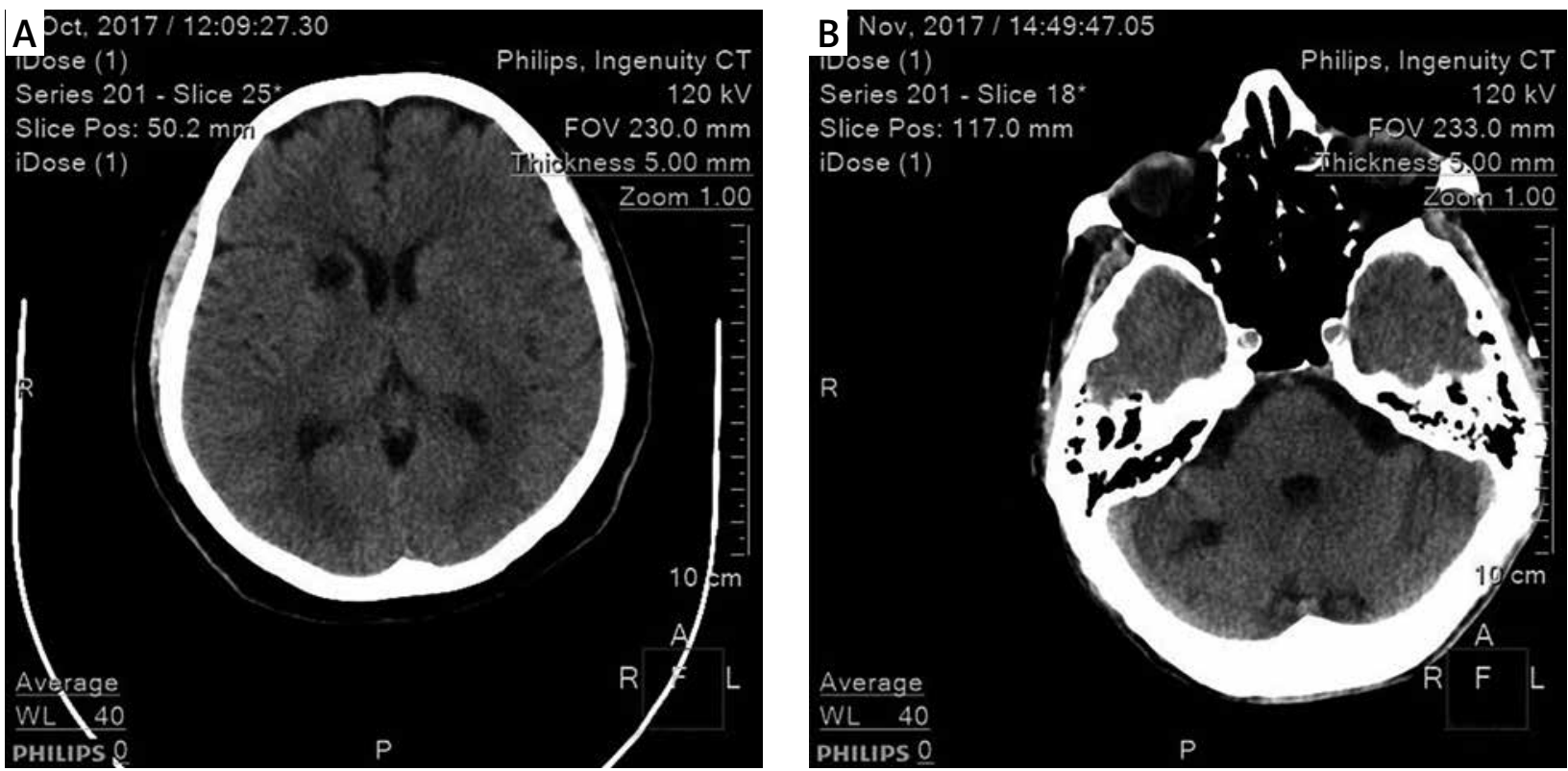

Figure 2. MSCT images showed lesion $\geq 3 \mathrm{~mm}$ separated by a sharp border from surrounding tissue in right basal ganglion (A) and right cerebellar hemisphere (B)

as mean \pm SD for continuous variables and percentages for categorical variables. The Shapiro-Wilk test was used to test normality and a $p>0.05$ was considered to indicate normally distributed data. Continuous variables that showed normal distribution were compared using Student's $t$ test, whereas the Mann-Whitney $U$ test was used for non-normally distributed samples. Categorical variables and frequencies were compared by means of the $\chi^{2}$ test. Statistical significance was defined as a $p<0.05$ for all comparisons. Parameters determining the developments of IBL were detected with the multivariate model using statistically significant parameters in univariate analysis. Multivariate logistic regression analyses were performed to determine significant predictors for IBL. Significant variables in univariate analysis at the level $p<$ 0.1 were entered in the logistic regression analysis.

\section{Results}

The MSCT was performed to patients within a mean of $3.52 \pm 2.14$ years after pacemaker implantation. In MSCT ex- 
amination silent IBL was detected in 21 (17.4\%) of 121 patients with CRT. Patients were divided into two groups, IBLS and normal CT scan. All parameters were compared and parameters predicting IBLs were found. When the device records of the remaining 121 patients were examined, SCAF was detected in 34 (28.1\%) patients.

When the demographic findings were compared between patients with and without IBL, it was found that patients with IBLS were older and had a higher frequency of hypertension ( $p<0.05$ for all). When the medical treatments of patients with IBLs were examined, it was found that the anti-platelet and anti-coagulant treat- ments did not have any statistical difference between the two groups ( $p>0.05$ for all). The $\mathrm{CHA}_{2} \mathrm{DS}_{2}$-VASc score was found to be significantly higher in the IBL group $(p<0.05)$ (Table I). Patients with IBL had a higher frequency of SCAF (Table II). It was found that the LVEF was significantly lower in patients with IBLs $(p<0.05)$. Other findings were similar between the two groups $(p>0.05$, for all) (Table I).

Ischemic brain lesion was found to be associated with age, $\mathrm{CHA}_{2} \mathrm{DS}_{2}$-VASc score, LVEF, higher frequency of hypertension and SCAF in univariate analysis (Table I). When the parameters found to be significant in univari-

Table I. Comparison of baseline characteristics of patients with IBL and normal CT

\begin{tabular}{|c|c|c|c|}
\hline Characteristics & $\begin{array}{l}\text { Ischemic brain lesions } \\
\qquad(n=21)\end{array}$ & $\begin{array}{l}\text { Normal CT scan } \\
\quad(n=100)\end{array}$ & $P$-value \\
\hline Age [years] & $69.3 \pm 5.9$ & $64.8 \pm 7.5$ & 0.011 \\
\hline Gender (female/male) & $8 / 13$ & $46 / 54$ & 0.325 \\
\hline Systolic blood pressure [mm Hg] & $127.6 \pm 14.4$ & $121.2 \pm 17.9$ & 0.125 \\
\hline Diastolic blood pressure [mm Hg] & $80.2 \pm 9.2$ & $78.7 \pm 10.6$ & 0.421 \\
\hline Pulse [beats/min] & $74.4 \pm 10.8$ & $78.6 \pm 12.3$ & 0.152 \\
\hline Body mass index $\left[\mathrm{kg} / \mathrm{m}^{2}\right]$ & $26.4 \pm 2.7$ & $26.6 \pm 3.8$ & 0.773 \\
\hline Smoking, $n(\%)$ & $2(9.5 \%)$ & $14(14 \%)$ & 0.439 \\
\hline Diabetes, $n(\%)$ & $11(52.4 \%)$ & $42(42 \%)$ & 0.276 \\
\hline Hypertension, $n$ (\%) & $16(76.2 \%)$ & $55(55 \%)$ & 0.048 \\
\hline Hyperlipidemia, $n$ (\%) & $11(52.4 \%)$ & $51(51 \%)$ & 0.382 \\
\hline Ischemic cardiomyopathy, $n$ (\%) & $9(42.9 \%)$ & $45(45 \%)$ & 0.512 \\
\hline Non-ischemic cardiomyopathy, $n$ (\%) & $12(57.1 \%)$ & $54(54 \%)$ & 0.512 \\
\hline Alcohol, $n(\%)$ & $7(33.3 \%)$ & $17(17 \%)$ & 0.087 \\
\hline NYHA (II/III/IV) & $9 / 10 / 2$ & $44 / 52 / 3$ & 0.556 \\
\hline $\mathrm{CHA}_{2} \mathrm{DS}_{2}$-VASc score & $3.81 \pm 1.43$ & $2.97 \pm 1.44$ & 0.017 \\
\hline HAS-BLEED score & $2.81 \pm 0.98$ & $2.93 \pm 1.12$ & 0.650 \\
\hline Basal QRS [msn] & $147.5 \pm 12.9$ & $147.5 \pm 13.0$ & 0.886 \\
\hline LBBB morphology, $n$ (\%) & $21(100 \%)$ & $86(94.5 \%)$ & 0.347 \\
\hline SCAF, $n(\%)$ & $11(52.4 \%)$ & $23(23 \%)$ & 0.009 \\
\hline $\mathrm{AF}<6 \min , n(\%)$ & $4(19 \%)$ & $21(21 \%)$ & 0.553 \\
\hline LV EF (\%) & $23.0 \pm 5.35$ & $26.8 \pm 4.64$ & 0.001 \\
\hline LA diameter $[\mathrm{mm}]$ & $35.5 \pm 4.3$ & $35.2 \pm 5.1$ & 0.972 \\
\hline Common carotid IMT [mm] & $1.17 \pm 0.21$ & $1.22 \pm 0.29$ & 0.523 \\
\hline Internal carotid IMT [mm] & $1.11 \pm 0.26$ & $1.19 \pm 0.34$ & 0.280 \\
\hline Anti-platelet treatment, $n(\%)$ & $10(47.6 \%)$ & $54(54 \%)$ & 0.367 \\
\hline Anticoagulation treatment, $n(\%)$ & $2(9.5 \%)$ & $6(6 \%)$ & 0.632 \\
\hline MSCT scan time after CRT [years] & $3.89 \pm 1.98$ & $3.45 \pm 2.18$ & 0.773 \\
\hline
\end{tabular}

$A F$ - atrial fibrillation, SCAF-subclinical AF, LV - left ventricular, $L A$ - left atrial, EF-ejection fraction, IMT - intima-media thickness. 
ate analysis were evaluated by regression analysis, presence of SCAF and LVEF were found to be independently associated with the risk of IBLS. According to this analysis, it was found that the presence of SCAF and every $1 \%$ decrease in LVEF increased the risk of IBL exposure by 3.5 times and $14.8 \%$, respectively (Table II).

\section{Discussion}

To our knowledge this is the first study that shows the relation of SCAF with silent IBLS. In our study, two important data related to the development of silent IBL in CRT patients were identified: (1) silent IBL incidence is $17.4 \%$ in our study population; (2) reduced LVEF and the presence of SCAF independently predict the occurrence of silent IBL.

The coexistence of AF and HF necessitates thromboembolic events, the most feared complications of both of these diseases. In the $\mathrm{CHA}_{2} \mathrm{DS}_{2}$-VASc scoring system that calculates the annual stroke risk of patients with $\mathrm{AF}$, patients with $\mathrm{HF}$ receive 1 point plus and are more likely to have a stroke. Because of these reasons, both clinical AF and SCAF diagnosis are of great importance in HF patients. In CRT implanted patients, continuous recordings of the atrial lead in place of conventional methods (Holter ECG etc.) and the clinician's ability to access these recordings allowed us to detect silent and asymptomatic AF. In patients with a cardiac electronic implantable device (CEID), atrial tachycardia episodes of $\geq 5$ or $6 \mathrm{~min}$ and $\geq 180$ beats/min were defined as AHRE [1]. In a study evaluating the relationship between IBL and AHRE in patients carrying cardiac devices, $\geq 5$ min AHRE was accepted as AF [5]. In a recent joint opinion report, asymptomatic episodes of AHRE $\geq 6 \mathrm{~min}$ and $<24 \mathrm{~h}$ recorded by CEID were defined as SCAF [9]. In our study, we identified the SCAF group as $\geq 6$ min AHRE in line with the European Heart Rhythm Association (EHRA) proposal and found that SCAF incidence was $28.1 \%$ in HF patients.

In patients with both conventional and ICD devices, documented AF, AHRE and SCAF were found to increase the incidence of thromboembolic events [10-12]. Similarly, in CRT patients, the presence of AHRE and AF increased the risk of stroke 2-5-fold [13-16]. In addition, patients with AHRE were found to have fewer echocardiographic responses to CRT, more ICD shocks, and more hospitalizations due to HF $[17,18]$. In the study of Shanmugam et al. [2], in CRT patients, thromboembolic events were similar between patients with initial permanent $A F$ and patients with AHRE detected by device monitoring. In the same study, the risk of thromboembolic events was 4 times higher in CRT patients who had AHRE in follow-up [2], Glotzer et al. [19] reported that the annual thromboembolism risk was 2.4 in patients with AHRE $>5.5 \mathrm{~h} /$ day in a 30-day follow-up. In a 2017 joint opinion report of the EHRA, OAC therapy was proposed in patients with AHRE
Table II. Multivariate regression analysis of ischemic brain lesions

\begin{tabular}{lccc} 
Characteristics & $\begin{array}{c}\text { Odds } \\
\text { ratio }\end{array}$ & $\begin{array}{c}\text { 95\% confidence } \\
\text { interval }\end{array}$ & $P$-value \\
\hline Presence of SCAF & 3.555 & $1.266-9.984$ & 0.016
\end{tabular}

$\geq 5.5 \mathrm{~h}$ and in patients with AHRE $>6 \mathrm{~min}$ if there is a very high risk factor [9].

In the literature, there were no studies examining the presence of subclinical IBL and associated parameters in HF patients with CRT. In patients with AF, MSCT and MRI are useful in symptomatic silent IBL detection [20-22] $M R I$ is superior to MSCT in assessing IBL. Except for the MRI-compatible battery, MRI is contraindicated in CRT patients. For this reason, we used MSCT for IBL evaluation. In a study of AF patients assessed by cerebral MRI, $40 \%$ of patients had one or more silent IBL [21]. Silent IBL is usually seen in patients with $\operatorname{SCAF}[22,23]$. The SCAF can be detected from both Holter and cardiac device records. A close association between the detected SCAF and IBL was found in both cases [5, 24]. Although there is a clear correlation between AHRE and increased stroke risk according to the obtained data, because of the highly subclinical nature of IBLs, the embolic risk is below that predicted. In a recent study of Benezet-Mazuecos et al. [5] there was an independent relationship between MSCT detected IBL and AHRE. In this study, AHRE's presence increased the IBL incidence three-fold. However, the SCAF rate may have been predicted higher than anticipated in this study, since AHRE > 5 min was accepted as SCAF unlike the current guideline recommendation of $\geq 6 \mathrm{~min}$. In our study, the close association between SCAF and IBL supports the results of the study of Benezet-Mazucos et al. However, in our study, we only evaluated HF patients with CRT. In addition, our study was more selective in the SCAF group because patients with AHRE $\geq 6$ min were accepted as having SCAF. According to our study results, the development of $I B L$ is increased 3.5-fold in patients with SCAF. In our study, we also found an independent association between reduced LVEF and IBL. Each 1 unit decrease in the LVEF was found to increase the risk of IBL by $14.8 \%$.

There are some limitations to our study. It is not correct to associate all IBLS with SCAF in patients with HF and CRT. Many stroke risk factors are present in these patients. As a single-center study, our patient cohort might be different from that in other centers. The sample size is relatively small and our results need to be confirmed in future large multi-center prospective trials. Currently, the efficacy and safety of new oral anticoagulations (NOAC) therapy in patients with AHRE/SCAF that are not clinically documented are unknown. Studies investigating the effect of apixaban and edoxaban treatment on thromboembolic prevention in patients with SCAF/AHRE detected by CEID are still in progress $[25,26]$. Although our study used MSCT for IBL 
detection, $M R I$ is more sensitive in IBL detection and even small infarcts can be detected. For this reason, the IBL frequency may be lower than expected.

\section{Conclusions}

According to our and previous studies, SCAF is independently associated with silent IBL presence. Patients with CRT should be closely monitored in terms of SCAF. Patients diagnosed with SCAF should be evaluated for $\mathrm{IBL}$ development and treated with the appropriate OAC.

\section{Acknowledgments}

This study was created at the Department of Cardiology and Radiology, University of Health Sciences - Adana Health Practices and Research Center.

\section{Conflict of interest}

The authors declare no conflict of interest.

\section{References}

1. Kirchhof P, Benussi S, Kotecha D, et al. 2016 ESC Guidelines for the management of atrial fibrillation developed in collaboration with EACTS. Eur Heart J 2016; 37: 2893-962.

2. Shanmugam N, Boerdlein A, Proff J, et al. Detection of atrial high-rate events by continuous home monitoring: clinical significance in the heart failure-cardiac resynchronization therapy population. Europace 2012; 14: 230-7.

3. Witt CT, Kronborg MB, Nohr EA, et al. Early detection of atrial high rate episodes predicts atrial fibrillation and thromboembolic events in patients with cardiac resynchronization therapy. Heart Rhythm 2015; 12: 2368-75.

4. Sade LE, Atar I, Özin B, et al. Determinants of new-onset atrial fibrillation in patients receiving CRT: mechanistic insights from speckle tracking imaging. JACC Cardiovasc Imaging 2016; 9: 99-111.

5. Benezet-Mazuecos J, Rubio JM, Iglesias JA, et al. Silent ischaemic brain lesions related to atrial high rate episodes in patients with cardiac implantable electronic devices. Europace 2015; 17: 364-9.

6. Santini M, Gasparini M, Landolina M, et al.; Cardiological centers participating in ClinicalService Project. Device-detected atrial tachyarrhythmias predict adverse outcome in real-world patients with implantable biventricular defibrillators. J Am Coll Cardiol 2011; 57: 167-72.

7. Kannel WB, Wolf PA, Benjamin EJ, Levy D. Prevalence, incidence, prognosis, and predisposing conditions for atrial fibrillation: population-based estimates. Am J Cardiol 1998; 82: 2N-9N.

8. Indik JH, Gimbel JR, Abe H, et al. 2017 HRS expert consensus statement on magnetic resonance imaging and radiation exposure in patients with cardiovascular implantable electronic devices. Heart Rhythm 2017; 14: e97-153.

9. Gorenek B, Bax J, Boriani G, et al. Device-detected subclinical atrial tachyarrhythmias: definition, implications and management - an European Heart Rhythm Association (EHRA) consensus document, endorsed by Heart Rhythm Society (HRS), Asia Pacific Heart Rhythm Society (APHRS) and Sociedad Latinoamericana de Estimulacion Cardıaca y Electrofisiologıa (SOLEACE). Europace 2017; 0: 1-23.
10. Defaye P, Dournaux F, Mouton E. Prevalence of supraventricular arrhythmias from the automated analysis of data stored in the DDD pacemakers of 617 patients: the AIDA study. The AIDA Multicenter Study Group. Automatic Interpretation for Diagnosis Assistance. Pacing Clin Electrophysiol 1998; 21: 250-5.

11. Gillis AM, Morck M. Atrial fibrillation after DDDR pacemaker implantation. J Cardiovasc Electrophysiol 2002; 13: 542-7.

12. Boriani G, Glotzer TV, Santini M, et al. Device-detected atrial fibrillation and risk for stroke: an analysis of $>10,000$ patients from the SOS AF project (Stroke preventiOn Strategies based on Atrial Fibrillation information from implanted devices). Eur Heart J 2014; 35: 508-16.

13. Abraham WT, Fisher WG, Smith AL, et al. Cardiac resynchronization in chronic heart failure. N Engl J Med 2002; 346: 1845-53.

14. Fung JW, Yu CM, Chan JY, et al. Effects of cardiac resynchronization therapy on incidence of atrial fibrillation in patients with poor left ventricular systolic function. Am J Cardiol 2005; 96: 728-31.

15. Hoppe UC, Casares JM, Eiskjaer H, et al. Effect of cardiac resynchronization on the incidence of atrial fibrillation in patients with severe heart failure. Circulation 2006; 114: 18-25.

16. Brambatti M, Connolly SJ, Gold MR, et al. ASSERT Investigators. Temporal relationship between subclinical atrial fibrillation and embolic events. Circulation 2014; 129: 2094-9.

17. Borleffs CJ, Ypenburg C, van Bommel RJ, et al. Clinical importance of new-onset atrial fibrillation after cardiac resynchronization therapy. Heart Rhythm 2009; 6: 305-10.

18. Leclercq C, Padeletti L, Cihak R, et al. Incidence of paroxysmal atrial tachycardias in patients treated with cardiac resynchronization therapy and continuously monitored by device diagnostics. Europace 2010; 12: 71-7.

19. Glotzer TV, Daoud EG, Wyse DG, et al. The relationship between daily atrial tachyarrhythmia burden from implantable device diagnostics and stroke risk: the TRENDS study. Circ Arrhythm Electrophysiol 2009; 2: 474-80.

20. Petersen P, Madsen EB, Brun B, et al. Silent cerebral infarction in chronic atrial fibrillation. Stroke 1987; 18: 1098-100.

21. Feinberg WN, Seeger JF, Carmody RF, Anderson DC, Hart RG, Pearce LA. Asymptomatic cerebral infarction in patients with atrial fibrillation. Circulation 1988; 19: 955-7.

22. Lin HJ, Wolf PA, Benjamin EJ, et al. Newly diagnosed atrial fibrillation and acute stroke: the Framingham Study. Stroke 1995; 26: 1527-30.

23. Ezekowitz MD, James KE, Nazarian SM, et al. Silent cerebral infarction in patients with non rheumatic atrial fibrillation. Circulation 1995; 92: 2178-82.

24. Marfella R, Sasso FC, Siniscalchi M, et al. Brief episodes of silent atrial fibrillation predict clinical vascular brain disease in type 2 diabetic patients. J Am Coll Cardiol 2013; 62: 525-30.

25. NCT01938248. Apixaban for the Reduction of Thrombo-Embolism in Patients with Device-Detected Sub-Clinical Atrial Fibrillation (ARTESiA); https://clinicaltrials.gov/ct2/show/ NCT01938248 (23 October 2015, date last accessed).

26. https://clinicaltrials.gov/ct2/show/NCT02618577. Non-vitamin $\mathrm{K}$ Antagonist Oral Anticoagulants in Patients with Atrial High Rate Episodes (NOAH). https:// clinicaltrialsgov/ct2/show/ NCT02618577 (22 December 2015, date last accessed). 